\title{
EL ESTATUS DEL TÍO MATERNO ENTRE LOS ANKAVE-ANGA: INICIACIONES MASCULINAS, CICLOS DE VIDA, GÉNERO Y PARENTESCO EN PAPÚA NUEVA-GUINEA
}

\author{
Pascale Bonnemère \\ Université de Provence - Francia
}

Resumen: Si nos atenemos a los estudios sobre las iniciaciones anga de Papúa Nueva-Guinea enfocados sólo en las actividades de los hombres, el objetivo de estos rituales era hacer crecer a los niños varones, endurecerlos en vista a posibles conflictos con las poblaciones vecinas e inculcarles los preceptos de la dominación masculina así como la legitimidad de su existencia. La etnografía de los Ankave-Anga permite una interpretación complementaria, ya que la eficacia de los rituales que marcan momentos claves de la vida masculina está garantizada por la participación de hombres y sobre todo de mujeres que pertenecen a categorias de parentescos muy especificas. El análisis de las acciones rituales que llevan a cabo estos personajes durante la iniciación de un hijo - de un hermano, de un sobrino uterino-, luego para el nacimiento de su primogénito y en fin en el momento de su muerte, revela otros aspectos fundamentales de estos rituales tales como el acceso al estatus de padre y de tío materno. Mostraremos pues que llegar a tal estatus conlleva una serie de transformaciones relacionales.

Palabras clave: parentesco materno, primer parto, rituales masculinos, transformaciones de relación.

Abstract: According to previous analyses of Anga initiations in Papua New Guinea, which are based on male activities only, the aim of these rituals was to make boys grow, to fortify them in a context of inter-tribal conflicts as well as to drum into them the rules of male domination and to legitimate its existence. The ethnography of the Ankave-Anga permits an alternative interpretation, however, since masculine life there is punctuated with rituals whose efficacy depends on men's participation but above all on the presence of some specific kinswomen. The analysis of the ritual actions performed by these characters when a son-a brother, a uterine nephew - is initiated, and then has his first child and finally when he dies reveals other major aspects of 
these rituals, such as reaching parenthood and maternal uncle status, which implies that a series of relational transformations has previously taken place.

Keywords: first birth, male rituals, maternal kin, relational transformations.

Hace mucho tiempo que los antropólogos que trabajan en Nueva-Guinea reconocieron la importancia otorgada al lazo de parentesco materno. Ésta se relacionó primero con la "familia bilateral como elemento focal en la red del parentesco" (Fortes, 1953, p. 33), en la que la filiación complementaria establece el lazo con los allegados del pariente que no determina la filiación. Pero al contrario de los modelos de filiación africanistas en los cuales "la filiación complementaria es el mecanismo principal por el cual se efectúa la segmentación dentro del linaje" (Fortes, 1953, p. 33), las situaciones que existen en Nueva-Guinea enseñan que los lazos por las mujeres no afectaban el mismo campo de lo social que los de la línea patrilineal (Barnes, 1962). Es cierto que le ofrecían a uno la oportunidad de cambiar de residencia, incluso de cultivar las tierras del grupo de su madre, pero tales ejemplos, numerosos, revelan más bien por una parte el grado de flexibilidad al que podía llegar la estructura de les organizaciones sociales de Nueva-Guinea donde la norma de filiación paterna sin embargo era predominante (incluso se calificó de "loose structure"), y por otra parte la importancia de otros criterios de pertenencia tales como el hecho de poder consumir comida proveniente de la tierra de un clan y compartirla con sus miembros lo cual contribuye progresivamente a transformar alguien de afuera en un miembro del clan (LiPuma, 1988, p. 100-101; Strathern, A., 1973, p. 33).

Los parientes maternos son esenciales en la vida de cada Ankave. A pesar del uso de un término de denominación específico para nombrarla, una tía materna es una verdadera segunda madre, lo que manifiestan constantemente las escenas de la vida cotidiana. Un lazo establecido del lado de la madre prevalece sobre el que puede reivindicar un padre sobre su progenitura. Así lo proclamaba una mujer durante un conflicto que se enconó cuando una pareja se estaba peleando porque su hija se había quedado en casa de la hermana menor de su madre más tiempo de lo previsto (Bonnemère, 1996, p. 144). En el mero medio de la altercación, la tía le negó violentamente a su cuñado el derecho de reclamar lo que fuera a propósito de su hija ya que ésta era descendiente 
de una matriz en todo punto similar a la suya. No se necesitó más para que se callara el padre que temía la mala influencia de esa mujer sobre su hija. Porque si bien los parientes maternos son figuras nutricias por excelencia, en cuanto están insatisfechos de las prestaciones, numerosas, que el padre de sus sobrinos y sobrinas así como su grupo les debe a lo largo de su vida, sus poderes benéficos se pueden convertir en poderes vectores de enfermedad y de muerte. ¡Cuántas veces escuchamos la preocupación de un padre al ver el adelgazamiento de su hijo o el retraso en la pubertad de su hija: "Tengo que darle todavía más a mi cuñado" o también "Debí de haber olvidado servirle al tío materno mayor antes que a los demás"! Esa serie de dones, que llevan nombres específicos, y esas creencias se relacionan con el hecho que los parientes maternos son los verdaderos "propietarios" - "owner" escribe Wagner (1967, p. 66-68) - de los niños que nacen.

Tales costumbres son corrientes en Nueva-Guinea. Seguramente fue Wagner quien describió primero y con más precisiones una situación similar a la que me tocó presenciar. Entre los Daribi del sureste de Nueva-Guinea, el tío materno es "just the same as mother" (Wagner, 1967, p. 66). Y puesto que proviene de la misma matriz que su hermana quien por ser madre les transmite su sangre a sus hijos, él está hecho de la misma sangre que sus sobrinos y sobrinas, distinta de la de sus hijos que heredan de la sangre de su madre. Así se desarrolla, igual que entre los Ankave, una teoría de la fabricación de los niños que establece el lazo con el tío materno (Wagner, 1967, p. 64). Esa comunidad de sangre, que funda el vínculode una substancia materna compartida, le da derechos al tío materno sobre los hijos de su hermana y justifica los dones frecuentes que recibe de las manos de su cuñado. Se trata de pagos de afiliación ("recruitment payments") que no cancelan para nada el lazo de consanguinidad de cada uno con sus parientes maternos y que sólo cumplen con los derechos que éstos tienen sobre uno en virtud de este lazo. Pues esta relación fundada sobre una sangre y una substancia compartidas permanece mientras sigan vivos los seres humanos que la componen (Wagner, 1967, p. 75).

Sin embargo ni entre los Daribi ni entre los Ankave nos encontramos en una sociedad matrilineal donde, por medio del tío materno, se asegura la afiliación de los hijos de su hermana al grupo de ésta. No es él quien transmite su nombre o su tierra a sus sobrinos y sobrinas. Aunque a veces esas dos situaciones dan esa impresión, las sociedades que las crean en realidad adoptaron normas de reclutamiento muy diferentes. Por eso, la aplicación de los modelos 
africanistas de filiación unilineal a las sociedades patrilineales des las Altas Tierras de Nueva-Guinea suscitó críticas repetidas y argumentadas desde la publicación del artículo de John Barnes (1962) que abrió esa vía pese a que él mismo no era especialista de Melanesia. En esta región de Oceanía casi nunca se da una correspondencia sistemática entre una entidad de clan y un grupo local. En un mismo caserío o en un mismo terreno, al lado de los descendientes en línea agnaticia de un ancestro particular se encuentran también los esposos y los hijos de sus hermanas sin que los primeros siquiera sean forzosamente mayoritarios. En Nueva-Guinea, principios de filiación complementaria se agregan al modo de descendencia reconocido (Barnes, 1962, p. 6) y permiten a los individuos reclamar el uso de un terreno cultivable o el acceso a un territorio de caza.

Los parientes maternos son pues personajes de primera importancia en la vida de los Ankave. Sin embargo tener una hermana con hijos no es bastante para hacer de un hombre un tío materno. Conseguir este estatus relacional (Théry; Bonnemère, 2008) implica pasar por una serie de rituales que se suelen llamar iniciaciones masculinas.

Estos rituales masculinos empiezan cuando un conjunto bastante numeroso de niños alcanza su primera década. Los maestros de iniciaciones deciden entonces perforarles el tabique nasal y someterles a una serie de pruebas físicas y mentales con el fin de hacerles crecer y, en el pasado de prepararlos también a combatir el enemigo. Las festividades empiezan con la construcción de dos grandes chozas colectivas localizadas una enfrente de otra a la orilla del pueblo. Una está destinada a las madres de los novicios y otra a sus padres. Las primeras vivirán recluidas de noche y de día en el transcurso del ritual mientras que los segundos podrán ir y venir entre el espacio forestal donde permanecerán los chicos, sus padrinos y los mayores de sexo masculino, y el espacio aldeano donde residen todos los demás habitantes del valle. Las ceremonias se organizan en tres fases, dos para el conjunto de chicos y la última en el momento del nacimiento del primogénito de un hombre. Las dos fases colectivas forman un conjunto que saca su nombre del principal acto ritual efectuado en sendos casos: "perforación del tabique nasal" y "unción de pandanus rojo". Los niños se quedan recluidos en el bosque durante el tiempo de la cicatrización de la herida nasal y luego, según las circunstancias, la segunda etapa empieza en seguida o después de un periodo que dura entre unos 
meses y un año; mientras tanto regresan a casa de sus padres (Bonnemère; Lemonnier, 2007, p. 147-175).

En vez de limitar estos rituales a una serie de acciones cuyo único fin es cambiar a los individuos nacidos de sexo masculino, el análisis que he hecho también toma en cuenta la totalidad de los gestos y prohibiciones que se les exige a determinadas categorías de personas relacionadas con él: en primer lugar a su madre, luego a su hermana y finalmente, durante la tercera y última fase, a su esposa. Al final de este largo proceso ese hombre se habrá convertido en padre pero el argumento defendido aquí es que sólo después de que su hermana se haya convertido en madre él alcanzará un grado de madurez y de inteligencia social dignas de su estatus de hombre adulto.

\section{Un largo itinerario masculino marcado por personajes femeninos}

Durante unas cuantas semanas que son las que dura su reclusión en el bosque, los novicios de las primeras y segundas fases sufren tratamientos corporales diversos: no sólo les perforan el septo sino que también reciben golpes de junquillo, se les estira los miembros y se les unta la piel con el zumo de determinadas frutas. Deben ayunar en varias ocasiones, y en otros momentos muy precisos, deben ingerir mixturas para calentarles o enfriarles el cuerpo que también está alternativamente confrontado a temperaturas extremas, por ejemplo acercándolo a un fuego inmenso y luego rociándolo con agua helada. Los novicios tienen que realizar esfuerzos físicos peligrosos tales como trepar a unos árboles de tronco ancho, cruzar un arroyo sobre un leño resbaladizo, pasar por unos corredores de ramajes angostos, o correr lo más rápido posible. Además están sometidos a pruebas psíquicas tales como la privación repetida de sueño, las amenazas de muerte, los engaños y la revelación de secretos que ocurren en un ambiente recargado de emociones de todas clases. Durante los rituales, la vida en común de los futuros iniciados implica un reparto de las tareas de leña y de agua, partidas de caza colectivas, así como una promiscuidad muy grande ya que toman las comidas juntos y duermen en una chozuela minúscula. Por otro lado les obligan a tener comportamientos y actitudes apremiantes: deben a menudo agacharse o sentarse en línea, extender las piernas y moverse sólo en fila india y únicamente cuando se lo ordenan. En otros momentos les piden que cierren la boca y aprieten los labios, o que agachen la 
cabeza y guarden silencio. También les enseñan lecciones de maneras diferentes. Directamente, cuando les explican la necesidad de compartir, de ayudarse mutuamente o cuando les enseñan las normas morales y los comportamientos adecuados de la edad adulta - no llorar, controlar su enojo, reprimirse, vencer su miedo - o las obligaciones y prohibiciones que conllevan determinadas ocasiones de su vida futura (no codiciar a la esposa de otro, no acercarse a las trampas de otro, etc.). Indirectamente, cuando asisten a representaciones en pantomima de acontecimientos de un pasado original o a rituales de peleas entre hombres de diferentes generaciones.

La iniciación masculina ankave consiste pues en una prueba que toma formas múltiples y cuyo objetivo explícito es endurecer a los chicos y así tenerlos preparados para una vida de hombre adulto que en un pasado reciente (hasta los años 1970) consistía principalmente en luchar para defender a los miembros de la tribu contra los ataques de los grupos enemigos. Pero también es un aprendizaje de las normas de conducta adecuadas y un lugar donde se expresan de manera metafórica y fragmentaria algunos fundamentos míticos de la cultura local. Se puede llegar a tales conclusiones con la pura observación de las actividades de los hombres en el bosque durante los rituales. Pero entre los Ankave - quizás se dé igualmente el caso en otra parte pero ya no es posible averiguarlo - los hombres no son los únicos en tener una actividad definida por el ritual durante las iniciaciones.

¿Qué nos enseña pues la observación simétrica de la actividad de las mujeres durante la iniciación de todos los chicos de una misma clase de edad? Para contestar a esta pregunta, es preciso considerar detalladamente las formas que toma esa implicación femenina en los rituales masculinos (Bonnemère, 2004) empezando por distinguir dos grandes categorías de mujeres: las hermanas mayores de los novicios por un lado y por otro sus madres que son testigos auditivos de la escena de la perforación del septo de sus hijos, de manera distinta y más señalada que sus hijas. Esa diferencia de postura, unas agazapadas debajo de sus capas de corteza y las otras sentadas con la mirada baja, permanece con diferentes formas durante todo el ritual. Mientras que las madres deben quedarse inmóviles o moverse sólo con el busto inclinado en la casa colectiva, las hermanas están de pie a sus alrededores y se van a los jardines para recolectar la comida común. El único traslado que deben efectuar las madres de los chicos se hace en fila india en un momento preciso del día para realizar un gesto muy específico. Del mismo modo los expertos rituales 
no ejercen acciones sobres ellas sino después de sentarlas en línea todas bien apretadas una contra la otra.

A quien vio lo que sucede en el bosque para los chicos le llama la atención la analogía de escenificaciones aquí en el pueblo y allá entre los árboles: un conjunto de seres poco individualizados que reciben el mismo tratamiento o efectúan los mismos gestos. Y cuando se compara lo que ocurre en ambos sitios al mismo tiempo, el paralelismo de las situaciones se revela especialmente significativo - no obstante las especificidades de cada grupo. En cambio nada tan sistemático une el conjunto de novicios al grupo de sus hermanas mayores.

La regularidad del paralelismo entre las acciones rituales de los novicios y las de sus madres se evidencia tanto mediante la naturaleza de esas acciones como por el momento en el que se realizan. Ambos grupos reciben capas de corteza batida hechas a propósito por los miembros de su parentela y entregadas por los expertos rituales. En el caso de las madres, la llevan por encima de la capa usada que ya vestían a diario; en el caso de los novicios, se trata de una prenda nueva que ponen encima de su cabeza privada de adornos. Desde el día que sigue la perforación del septo, madres e hijos acuden cada día al amanecer a orillas del arroyo más cercano a su espacio de reclusión respectivo y remojan esa capa nueva en el agua antes de ponérsela de nuevo sin escurrirla y regresan a sus chozas. Lo que dicen las mujeres acerca de este gesto remite a la infancia de los novicios, un período de su vida en el que temían la lluvia y eran desobedientes. En cuanto al hecho de llevar ellas la capa, tampoco suscita ninguna interpretación elaborada por su parte. Sólo dicen que cuando la llevan puesta, piensan en sus hijos y se quedan quietas para que la herida nasal de la perforación del septo llegue a cicatrizar correctamente ya que el más mínimo gesto puede impedir o diferir este proceso que se efectúa en varias etapas, cada una con cambio de la barra nasal y suspensión de algunas prohibiciones.

Las madres, las hermanas mayores y los novicios están en efecto unidos en el respeto de una serie de actitudes, algunas de tipo alimentario. Desde el inicio de los rituales, esas tres categorías de personas reciben, cada una por su parte, una mixtura a base de sal vegetal cuya composición varía ligeramente pero cuyo resultado es similar: hacer que suba la temperatura del cuerpo. Más precisamente se trata de calentarle el hígado al novicio iniciando de esa manera su muerte simbólica a la vez que se borra en el vientre de la madre toda huella del nacimiento del chico cuyo destino está ahora entre las manos de los expertos rituales. Luego, madres y novicios reciben exclusivamente azúcar de 
caña lo que esta vez provoca un enfriamiento de su cuerpo. Llega entonces el momento de imponer nuevas prohibiciones. El zumo de fruta de pandanus roja, la nuez de areca, ${ }^{1}$ el agua, algunas verduras (las que se vuelven viscosas después de cocerlas) y algunas variedades de tubérculos están prohibidos para las madres, las hermanas mayores y los novicios a partir del momento en el que se perfora el septo. El zumo de fruta de pandanus es un alimento apreciado que se obtiene cociendo la fruta grande de este árbol y exprimiendo sus semillas. La mayoría de las veces se consume como salsa que le echan a los boniatos o a los taros para las grandes comidas colectivas. Por ser considerado como substituto de la sangre, se prohibe su consumo en determinados momentos del ciclo de vida o por el contrario se recomienda fuertemente en otros, por ejemplo cuando alguien está enfermo. También es la principal comida de las mujeres embarazadas porque se suma a la sangre que tiene en su cuerpo, de la que se alimenta el feto.

Se levantan las últimas restricciones conforme van cambiando las barras nasales (tres en total) excepto las que se aplican al pandanus rojo y a la nuez de areca; éstas siguen vigentes hasta la comida colectiva del final de las ceremonias. Poner término a esas dos prohibiciones mayores - por prolongadas - se lleva a cabo mediante una gran escenificación ritual en la que resalta de nuevo la diferencia de tratamiento entre las madres y las hermanas. En cada caso, el procedimiento empieza por el enfriamiento con agua que se vierte en la coronilla con un continente de bambú.

Los expertos rituales entregan a las madres las dos nueces de areca que levantan el tabú mientras que las hermanas las reciben de las manos de sus hermanos ya iniciados. Éstas serán las últimas en desprenderse de las nueces, al final del todo de las ceremonias, cuando los hombres y los chicos ya regresen al pueblo. Por otro lado, ellas no habían sido friccionadas con tierra durante el último cambio de barra nasal a diferencia de las madres. El conjunto de esas diferencias pone de relieve la implicación especial de las madres durante la iniciación de sus hijos.

Durante el periodo de reclusión estricta que corresponde a la etapa de cicatrización de la herida nasal de los chicos, las madres no sólo evitan comer

\footnotetext{
1 Consumida con una hoja o un amento del árbol Piper betle y cal, la nuez de areca de la palmera de betel es un principal constituyente del masticatorio que produce una masa liquida roja. Según mis análisis, se asocia este producto a la sangre de los cadáveres (Bonnemère, 1996, p. 263-265).
} 
determinados alimentos sino que, sometidas también en este caso a las mismas prohibiciones que sus hijos, se abstienen de tocarse la piel con las uñas o de rascar la capa negra de los tubérculos cocidos para ellas debajo de las cenizas por las demás mujeres. Igualmente, cuando escuchan el canto de un pájaro de mal agüero o el zumbido de un insecto, sobre todo de una mosca, madres e hijos dejan inmediatamente de masticar la comida que tienen en la boca y a menudo la escupen. Esos volátiles están asociados a la putrefacción y a la muerte así que se debe aniquilar sus manifestaciones en el momento en que los principales participantes del ritual ingieren algún alimento para evitar que esos vectores de muerte se vayan a introducir en sus cuerpos por medio de la comida. En fin las cortezas de las cañas de azúcar que deben consumir madres e hijos en momentos especiales del ritual son recogidas por los expertos rituales en los dos espacios, o sea respectivamente el pueblo y el bosque; luego éstos irán a esconderlas en lugares que sólo ellos conocen.

Cuando ya se ha acabado la cicatrización de la herida nasal, las mujeres de todas las categorías hacen algunas prendas y adornos que llevarán los iniciados cuando vuelvan al pueblo. Esa preparación tarda unos quince días mientras los chicos van a cazar colectivamente y siguen sufriendo varias pruebas para endurecerlos. El día señalado para la salida de la reclusión, se cuece en un horno grande semienterrado la comida que recogieron las mujeres, exceptuando las madres. Dos mujeres con experiencia y miembros de dos clanes diferentes primero trajeron hojas de keringi'y tierra amarilla anaranjada proveniente de la fuente de un río. Estas hojas sirven para envolver un boniato y un taro Colocasia antes de ponerlos en el centro del horno; ambos alimentos se destinan a cada uno de los iniciados,. Durante la preparación del estofado, los chicos bajan en fila india del bosque ricamente ataviados y escoltados por sus padrinos respectivos, quienes son iniciados de la cohorte anterior que los acompañan a lo largo de las pruebas. Cuando llegan a la linde del caserío, las dos mujeres experimentadas los esperan una enfrente de otra y les frotan la cabeza y el busto con tierra que ellas mimas trajeron. Acompañado de una fórmula, este gesto es en todo punto similar al que efectúan las madres sobre el cuerpo de su recién nacido.

Cuando ya pasaron por las manos de esas dos mujeres, los iniciados se sientan juntos en un rincón apartado del pueblo mientras siguen cociéndose los tubérculos. Cuando la cocción llega a su fin, las mujeres se reúnen alrededor del horno, agarran un paquete envuelto en hoja de keringi', lo ponen 
sobre su capa de corteza y cada una llama por su nombre a su hijo. Los chicos desfilan uno tras otro, ofrecen a su madre un pájaro o una pequeña rata del campo que cogieron en el bosque y ahumaron, agarran la capa de corteza que contiene el paquete de comida y luego se alejan para reunirse aparte. Después de consumir los alimentos, devuelven la capa y la hoja de keringi' a sus madres que parecen apesadumbradas de la transformación radical sufrida por sus hijos. Se juntan las hojas y las dos mujeres experimentadas las llevan de vuelta donde las cogieron de manera muy discreta. Luego la vida vuelve a su cauce, los padres de los iniciados regresan a su hogar con sus hijos, incluidos los que acaban de sufrir las pruebas iniciáticas.

Unos años después, los chicos ya se han convertido en hombres casados y un día se anuncia la próxima llegada de su primer hijo. Comienzan entonces los gestos y comportamientos definidos por el ritual.

La nuera, que no vive lejos con el hijo, les comunica la noticia a sus suegros; ellos son quienes le dicen al futuro padre que su esposa está embarazada y organizan unos días después una gran cena con ayuda de sus consuegros. Es el momento oportuno para decirle al joven que de ahora en adelante tendrá que dejar de consumir zumo de fruta de pandanus rojo, carne de animales cazados y de beber agua. Tampoco puede seguir haciendo las tareas masculinas tales como la confección de nudos, por miedo a que el bebé se quede atrapado dentro de la matriz materna. Esta prohibición le impide construir casas y barreras o hacer arcos, flechas y trampas. Además, en cuanto se sabe la noticia del embarazo, una hermana del esposo - en el caso ideal, mayor que él pero todavía sin hijos - también deja de tomar zumo de fruta de pandanus rojo.

Los padres también le dicen a la joven pareja que prepare una estera especial llamada ogidze, de corteza batida, tinta con zumo de fruta de pandanus rojo, secada al sol; luego se enrolla y queda envuelta hasta que se use varios meses después. Tienen que confeccionarse más manteles de corteza batida, pero son ordinarios y se hacen más rápidamente: dos son para el futuro padre (llevará el primero colgado de la coronilla y el segundo, llamado iZiare, anudado alrededor de su cuello le cubrirá las nalgas); se regala otro a su hermana y otro más a la futura madre. También se debe preparar algo de cal para poder mascar el betel después del nacimiento y para intercambiarlo contra sal vegetal con los vecinos Iqwaye.

Cuando las capas de corteza batida ordinarias están listas, el experto del ritual Sewange' le cubre la cabeza al joven en el bosque, siguiendo un 
protocolo especial. En cambio la mujer embarazada puede ponerse ella misma su capa. Para un hombre llevar tal capa es tan poco habitual que cada uno sabe que espera a su primer hijo. No podrá quitársela antes que su esposa dé a luz. Su silueta especial también indica que se encuentra en estado de tabú y que se abstiene más especialmente de zumo de pandanus rojo. Como lo dijimos, su esposa puede y hasta debe consumir este alimento en grandes cantidades, ya que su hijo se alimenta de tal zumo y fabrica su sangre a partir de éste.

Entonces, durante todo el tiempo de la gestación, tres personas se hallan unidas por el hecho que cada una recibió una nueva capa de corteza que debe llevar de la misma manera y durante el mismo tiempo. Forman una tríada unida por las actitudes y las prohibiciones que dos de ellos respetan más especialmente: el futuro padre y su hermana. Esta situación recuerda las costumbres definidas por la antropología social con el término de "couvade". Desde 1910, Frazer marcaba la diferencia entre dos tipos distintos de tal fenómeno:

One of these customs consists of a strict diet and regimen observed by a father for the benefit of his newborn child, because the father is believed to be united to the child by such an intimate bond of physical sympathy that all his acts affect and may hurt or kill the tender infant. The other custom consists of a simulation of childbirth by a man, generally perhaps by the husband, practised for the benefit of the real mother, in order to relieve her of her pains by transferring them to the pretended mother. (Frazer, 1910, p. 254).

Los autores antiguos asimilaban tales tabúes a una forma de magia simpática basada en la creencia que se transmite a los futuros padres las características del animal o de la planta consumida. Respetar las prohibiciones alimentarias era pues una medida para proteger al bebé. La mayoría de las interpretaciones coincidían en que la couvade era un rito de paternidad y que su presencia era más probable en las sociedades donde la relación matrimonial era más frágil (Douglas, 1968, p. 24) o en regímenes de bienes matrilineales (Malinowski, 1927, p. 192). Según estos autores, al respetar los tabúes y adoptar un comportamiento especial, el papel del padre en la procreación se veía legitimado y le permitía establecer sus derechos sobre sus hijos. ${ }^{2}$ Esta visión

2 Diremos que el pago de la compensación matrimonial al grupo de la esposa es otra costumbre que establece los derechos del esposo sobre la progenitura de la pareja. 
ha sido criticada en publicaciones más recientes, por ejemplo las de Patrick Menget (1979, p. 259) que afirma que los derechos de un padre sobre sus hijos le vienen del matrimonio y no de teorías de la concepción o de costumbres relacionadas con el nacimiento. En un artículo famoso, Peter Rivière (1974, p. $431,433)$ defendió la idea que los actos de un futuro padre no tienen relación con la buena salud del bebé sino con su existencia espiritual aunque el modelo sea el de la fisiología humana. Así, la couvade tiene que ver con la creación de una persona completa, compuesta de un cuerpo y de un espíritu. Según este autor se trata de una de las numerosas instituciones que tratan del problema - que él considera universal - de la doble composición de los seres humanos (Rivière, 1974, p. 434).

También se han propuesto interpretaciones psicológicas que caracterizan la couvade como una manifestación de la identificación con los miembros de sexo opuesto, que sería necesaria para compensar la presencia tenue de los padres y la proximidad fuerte con las madres durante los primeros años de vida (Munroe; Munroe; Whiting, 1973). Es de notar que todas estas hipótesis ponen al padre en estado de "couvade" en una relación de identificación con su esposa en el momento del nacimiento de su hijo (Doja, 2005, p. 925-926). ${ }^{3}$ En un artículo enteramente dedicado a las costumbres de couvade entre los Bimin-Kuskusmin de Papúa Nueva-Guinea, Fitz Poole (1982, p. 87) revela que para los miembros de esta sociedad, "los síntomas de couvade están reconocidos como indicios de enfermedad a la vez generales y particulares relacionadas con el nacimiento, y el ritual que corresponde da un modelo cultural de (a nivel cognitivo) y para (a nivel terapéutico) tales enfermedades".

Entre los Ankave, no se representa al futuro padre como especialmente vulnerable y, en la medida en que no es el único en estar en situación de tabú, la couvade no puede calificarse de rito de paternidad. Más bien se trata, tal como lo propone Laura Rival (1998, p. 631) para los Huaroni de Amazonia, de un rito de coparentalidad. Además las prohibiciones que tiene que respetar la futura madre deben analizarse conjuntamente con las que les tocan al futuro padre y a su hermana. El futuro padre y su hermana se abstienen de consumir alimentos que se volverían sangre y causarían una hemorragia en la

3 Sin embargo, Lévi-Strauss (1962, p. 258-259) desaprueba tal afirmación: según él, el padre no imita a su esposa que da a luz sino más bien al hijo.

Horizontes Antropológicos, Porto Alegre, ano 16, n. 34, p. 405-426, jul./dez. 2010 
parturienta; tampoco efectúan tareas que impedirían la salida del feto ${ }^{4}$. La mujer embarazada no come carne de animales con determinados caracteres (tales como un pelaje manchado) o costumbres (vivir en anfractuosidades del suelo como los marsupiales, colocarse debajo de las piedras como ranas y anguilas, establecerse en los bambús como los insectos etc.) que podrían transmitirse al hijo. Las plantas con tallo sinuoso u hojas vellosas también están proscritas; las primeras por miedo a que el niño se quede bloqueado en la matriz y las segundas porque la piel del bebé se cubriría de llagas.

En todos los casos, se trata de "prohibiciones para el prójimo", una norma muy operante entre los Ankave y que forma parte de un sistema de pensamiento en el cual gozar de buena salud depende más - o por lo menos en igual medida - de las acciones de los demás que de las efectuadas por uno mismo. ${ }^{5}$ En suma, el hecho que los dos padres deben respetar prohibiciones nos lleva a proponer lo siguiente: el ritual de primer nacimiento no sólo es la tercera y última etapa de las iniciaciones masculinas sino también y sobre todo el momento en que se establece una nueva pareja parental con asistencia en el ritual de la hermana del futuro padre. Ésta actúa probablemente en calidad tanto de hermana como de persona representante de la patrilínea del recién nacido.

De manera más general, nuestra propuesta es que la couvade debe analizarse como un elemento perteneciente a un conjunto de prácticas vinculadas a momentos claves del ciclo de la vida. Entre los Ankave las prohibiciones alimentarias y del comportamiento se imponen en varias ocasiones, lo que nos impide considerar la couvade como una institución que podría analizarse aisladamente y que sería distinta de las iniciaciones masculinas o de las ceremonias mortuorias, por ejemplo que implican conductas similares.

Sin exponer con todo detalle los gestos prodigados en el nacimiento propiamente dicho, como ya se hizo (Bonnemère, 2009), cabe recordar que la que anuncia el nacimiento de un primer hijo es la mujer que ayudó en el parto y que, al poco tiempo, un nuevo periodo de gestos rituales y de normas de

4 Para darse cuenta de lo que son las prácticas de parto y los temores que suscita en esta región del mundo, referirse a Fiti-Sinclair (2002).

5 Aún si Marilyn Strathern (1988) no consideró en detalle las prohibiciones alimentarias en The gender of the gift, su argumento sobre la persona melanesia siendo el producto de relaciones múltiples es obviamente pertinente para entender la situación ankave de la gente absteniéndose de alimentos específicos para actuar sobre el cuerpo de alguien más.

Horizontes Antropológicos, Porto Alegre, ano 16, n. 34, p. 405-426, jul./dez. 2010 
comportamiento empieza para las personas que ya habían sido involucradas en el embarazo.

Primero, una dieta estricta prohíbe el agua como bebida, el zumo de fruta de pandanus rojo y el betel a los dos nuevos padres durante tres días después del parto. Pero no son los únicos en respetar esas prohibiciones ya que el hombre que, unos días después de la caza colectiva de marsupiales, llevará al nuevo padre al bosque para la etapa secreta del ritual Sewange', así como el esposo de su hermana, también deberán someterse a tales prohibiciones. En cuanto a la nueva madre, la única comida que puede - y en realidad debe ingerir es zumo de caña porque hace que suba la leche. Los Ankave piensan que el calostro es un alimento nefasto para el bebé y que sólo se convierte en leche cuando la madre ha absorbido este zumo azucarado.

Después de los tres días de dieta, el experto ritual reúne tres variedades especiales de cañas de azúcar y las somete a un tratamiento mágico antes de dárselas al nuevo padre. Será su única comida durante dos días más. Luego, el experto recoge dos variedades especiales de taros Colocasia (a'we imema'we y wionge') y le pide a su esposa que los cueza con un boniato amene'ke. Cuando ya están cocidos, el especialista efectúa gestos mágicos, los trocea y los lleva al bosque. Allí, le dice al nuevo padre que se coma la mitad; entonces regresan juntos al pueblo.

La esposa del experto ritual toma la segunda parte de los tubérculos cocidos y los trae a la madre que descansa en una chozuela construida especialmente para ella. A los tubérculos les agrega una mixtura hecha con jengibre, hojas con olor a limón y verduras sobre cuyas hojas se ha echado sal. Se dice que esta comida fortalece el útero.

Mientras tanto los hombres van de caza a los marsupiales durante varios días, el tiempo necesario para capturar los animales suficientes para que cada mujer en el pueblo (excepto unas categorías de parientes que no los pueden comer, lo explicaremos luego) reciba un pequeño pedazo de carne. Los marsupiales prisioneros de las trampas que el esposo había colocado en el territorio de su clan se regalan primero a su esposa. Luego llega el momento de organizar el ritual Sewange' que tarda un día entero y se divide en dos etapas: una secreta para el sexo opuesto así que hombres y mujeres lo preparan por separado desde el amanecer hasta el mediodía y la otra, pública, que tiene lugar por la tarde. 
Poco antes del amanecer del día señalado (por lo general entre cuatro y ocho días después del nacimiento) y mientras el nuevo padre está todavía en el bosque con uno (o dos) joven (jóvenes) que haya(n) vivido el mismo acontecimiento, varias mujeres, entre ellas la esposa del acompañante, su propia hermana mayor, la hermana de su esposo y su madre, visitan a la nueva madre en su choza. Entonces se destruye esta habitación siguiendo un modo operativo que difiere según el sexo del bebé: para un niño, las mujeres susurran un canto femenino que suele entonarse durante las iniciaciones; si es niña, se ríen a carcajadas y así expresan su alegría al recibir un nuevo miembro de su sexo. Al mismo tiempo, otras mujeres allegadas a la nueva madre van pelando tubérculos para la gran cena que marcará el final de las actividades rituales.

La madre agarra su nueva falda de fibras de corteza batida y pronto llevará la capa que le preparó su esposo durante el embarazo. Las mujeres que asistieron a la destrucción de la choza van bajando el camino que va al gran río Suowi. Allá, la madre deja a su niño de pecho sobre una capa de corteza y se sienta en el agua. Su hermana le fricciona el cuerpo con hojas olorosas. Cuando la nueva madre ya se ha bañado y vestido con prendas nuevas, todas las mujeres regresan al pueblo y se instalan cerca de la plaza donde se realizará la etapa pública del ritual. La madre, su hermana, la hermana de su esposo y la esposa del compañero de su esposo en el bosque se esconden detrás de hierbas altas, ocultas a las miradas. Varias horas seguidas, platican sigilosamente y se cortan el pelo. La hermana de la nueva madre se encarga de cortárselo a ella mientras otras mujeres le dibujan cortas líneas rojas en la frente con semillas de urucú (Bixa orellana). Hay niños presentes y cada uno descansa debajo de los árboles.

Antes del amanecer, ya se había empezado a escuchar las bramaderas y se siguen tocando de manera intermitente; señalan la presencia de entidades sobrenaturales poderosas. Supuestamente las mujeres interpretan el sonido de esas tablillas de madera que los hombres hacen girar en el aire como la manifestación de espíritus silvestres malévolos y no miran hacia el lugar de donde proviene el ruido. Es difícil averiguar lo que saben exactamente pero de todos modos ninguna se avendría a contradecir las afirmaciones de los hombres. Mientras se escuchan los que Tuzin describió como "numinous objects", el nuevo padre se va al bosque con el hombre o los hombres que vivieron el mismo evento hace poco. Le dicen que recoja nueces de areca y le enseñan cómo recoger esa tierra de color rojo anaranjada que necesitará dentro de poco. 
Entonces los dos o tres hombres van bajando hacia el lugar donde, desde varias generaciones, los hombres ankave efectúan el ritual Sewange’ porque allí se encuentran varios árboles con hojas que enrojecen y caen (Elaeocarpus $s p$.). En este preciso lugar, el experto ritual ha plantado unas cordilinas rojas y extendido el mantel de corteza ogidZe sobre una estructura de madera. Encima ha colocado con esmero los adornos de conchas y plumas y también los talabartes trenzados. Poco antes de la llegada de los hombres, el experto se esconde debajo del mantel ogidze, agarra dos bejucos y los manipula para que se mueva el mantel. La escena es la visión materializada de un ancestro primordial. El mito relata que su cadáver dio origen a las cordilinas de hojas rojas y al pandanus del mismo color y su sangre produjo la tierra roja anaranjada. De una manera $u$ otra, se usan todos estos materiales durante todas las etapas de las iniciaciones (Bonnemère, 1998, p. 89-95).

Cuando ya está listo el experto, los hombres, numerosos, esperan a los demás que van bajando de la parte alta del bosque, con un hacha o un palo en la mano. El ritual secreto para el nuevo padre consiste en unos golpes asestados en los hombros y las mejillas por el experto que tiene en la mano una cordilina y un plantón de jengibre. Tratan igual a sus compañeros que escuchan una vez más las palabras que le dicen al nuevo padre. Son esencialmente normas de conducta: "no debes robar los bienes ajenos, no debes entrar en los jardines que pertenecen a otros sin su permiso, no debes tomar la esposa de otro hombre", etc. El nuevo padre se reúne entonces con el experto ritual debajo del mantel ogidze y cerca del fuego donde se le calientan las articulaciones. Luego aprende cómo quitar el tabú de tres días sobre la mascada de betel después del nacimiento, algo que tendrá que repetir tras el nacimiento de cada uno de sus hijos.

Luego viste las prendas nuevas y se pone los adornos expuestos en el ogidze. Uno de los hombres corta el mantel de corteza roja en tiras largas y las distribuye a todos los hombres que las llevan ceñidas en la frente. Esas tiras de corteza serán reunidas después del ritual y entregadas a la madre de un niño de tres años para hacerle su primer taparrabo. Entonces les cortan la barba y el pelo a los tres principales protagonistas y el nuevo padre luce un adorno de cabeza especial hecho de plumas y llamado Sewange'. Se dice que están ya fortalecidos y listos para regresar al pueblo. El experto termina el ritual secreto masculino dándole a cada uno de los hombres una cordilina roja para plantar en su propio jardín. 
El nuevo padre lleva una larga estaca me'wa que le cortó uno de sus compañeros. En una extremidad están colgados los collares de concha y los talabartes de fibras trenzadas destinados a su esposa. Mientras los hombres van bajando despacio de la colina, hacia el mediodía, sus madres y sus hermanas lloran pues se han vuelto hombres adultos y ya empiezan el declive de su vida. Una anciana experimentada les fricciona el cuerpo con la misma tierra amarilla anaranjada que se usa al final de cada etapa del ritual y pronuncia en voz baja las mismas palabras.

La nueva madre, la esposa del compañero de su marido y la hermana de éste se acercan agachadas o con el busto inclinado tras salir de su escondrijo. No se dice nada preciso a propósito de esta postura: según algunas mujeres, evoca su posición en el transcurso de la reclusión durante las etapas colectivas de las iniciaciones, cuando no se pueden poner de pie y se pasan la mayor parte del tiempo sentadas o agachadas dentro de la casa grande construida con motivo del ritual. En opinión de la antropóloga, recuerda la postura del casuario, un ave que según el mito resulta de la transformación de una mujer que no se portaba como una buena esposa (Bonnemère, 1996, p. 325-328).

El experto agarra la estaca me'wa y le da los adornos a la nueva madre. $\mathrm{Su}$ esposo u otro hombre hiende la estaca en pedazos largos y delgados y los clava en el suelo formando un círculo y dejando los trozos más pequeños en el centro. Entonces el experto le prende fuego a este dispositivo con una antorcha de bambú. Luego ofrece algunas nueces de areca a la esposa y a la hermana del marido que está sentada al lado de ella. Las dos levantan el tabú sobre la mascada del betel escupiendo el primer zumo en el fuego. Los marsupiales cazados por los hombres son cocidos en una corteza de árbol o en un horno semienterrado tras pasar su pelaje por las llamas del fuego me'wa. Las dos hermanas de los padres del recién nacido preparan uno de estos animales, a ser posible un tse' arma' - porque crece rápido. Se mezclan las cenizas del pelaje quemado con tierra amarilla anaranjada y agua y la abuela materna del bebé le fricciona el cuerpo con esa mezcla. Entonces se pasa rápido al bebé por encima de las llanas mientras una anciana pronuncia una fórmula mágica. Luego tratan igual a todos los bebés alrededor para "calentarles los huesos" según los Ankave.

Las dos hermanas trocean el tse' arma', ellas no lo comen y la totalidad de la carne es para la madre del bebé. Tampoco pueden recibir otro de los marsupiales que se reparten entre las mujeres y sus hijos jóvenes. Para la hermana 
y la madre del esposo, el consumo de estos animales equivaldría a cometer un acto de canibalismo hacia su hermano o su hijo con quien comparten la misma sangre. El día se acaba con la cena de taros y boniatos, preparados en gran cantidad desde la mañana, en la que, ahora sí, todos pueden participar.

\section{Nació una pareja parental}

Hemos descrito las diferentes etapas que cambian un chico joven en padre, pero más allá del personaje masculino sobre quien, al parecer, se enfoca el ritual, la participación de varias categorías de personas revela que se trata sobre todo de convertir una pareja casada en unos padres a los que se transmiten conocimientos especializados acerca del recebimiento de los recién nacidos. El análisis de las prohibiciones que respetan conjuntamente con determinadas mujeres, y de los gestos efectuados en el mismo momento en unas zonas rituales geográficamente separadas pero unidas en el pensamiento y en los actos, saca a luz un sistema de representaciones en el que resalta un modo operativo propio de esta sociedad: la capacidad de actuar para el prójimo. Sin embargo esta capacidad parece más espontáneamente vinculada a las mujeres que a los hombres. La descripción que antecede de los comportamientos rituales durante las etapas colectivas y del ritual de primer nacimiento es altamente significativa al respecto: sólo las mujeres están en posición de actuar para el prójimo, y son hombres, hijos y hermanos menores más particularmente, quienes se benefician con las acciones del prójimo, que ganan autonomía y crecen al transformar las relaciones que tienen con los personajes participantes del ritual. Pues el resultado de las primeras etapas colectivas es efectivamente la transformación de una relación de tipo simbiótico entre una madre y su hijo, cuyo modelo es la gestación con la idea que el feto se alimenta de la sangre de su madre; el gesto que sigue el regreso de los chicos al pueblo es emblemático del contenido de la nueva relación establecida hasta el final de sus vidas: un don de caza menor (aves, campañoles) contra tubérculos. Madre e hijo han pasado de una relación de fusión a otra en la que ya se ha hecho posible el intercambio recíproco. En las etapas rituales ulteriores, la madre ya no interviene concretamente sino respetando algunas prohibiciones, esencialmente sobre los animales cazados por su hijo y los que regala repetidamente el grupo del futuro esposo de su hija. En cambio la hermana, que en el caso ideal 
todavía está sin hijos, esta vez sí es omnipresente. Su intervención empieza en la primera etapa de las iniciaciones, pero en menor grado pues, al contrario de la madre, la hermana no vive reclusa ni se queda inactiva. Sin embargo su implicación es esencial ya que hace la transición entre dos personajes centrales de la vida de un hombre: la que le dio a luz y la que traerá sus propios hijos al mundo. Ya lo vimos, interviene especialmente cuando su hermano está a punto de ser padre de un primer hijo, adoptando la misma silueta y respetando la misma prohibición de fruta de pandanus rojo. Después de nacer el bebé, ella será una compañera de la nueva madre durante las etapas secretas y públicas del ritual. Al compartir esos momentos de vida íntima con su hermano, le permite acceder al estatus de padre.

Luego, en la última etapa de acceso a la edad adulta, le permitirá ser un tío materno cuando ella se vuelva madre a su vez. Ya vimos la importancia de este estatus en la sociedad ankave. Ser un tío materno significa ser capaz de actuar para el prójimo. Para un hombre, hasta es la única posición que le ofrece esta oportunidad y vimos que no sólo tiene aspectos positivos dado que el tío materno puede en situaciones conflictivas actualizar poderes vectores de enfermedad, de adelgazamiento o de muerte en contra de los sobrinos y sobrinas uterinos.

Mientras que una mujer tiene esta capacidad de actuar para el prójimo para transformar una configuración relacional incluso antes de dar a luz a su primer hijo, el hombre debe conseguirla en el transcurso de un camino largo y progresivo en el que intervienen varios personajes femeninos. Casi se podría decir que una mujer se encuentra en la posición de actuar para el prójimo por una calidad propia que no precisa adquirirse, probablemente la de ser una madre potencial. El hombre primero debe demostrar que puede ser padre, pero no es suficiente ya que su verdadera aptitud a tener influencia sobre el cuerpo de otra persona sólo se manifiesta de verdad cuando su hermana se vuelve madre. Para los Ankave, la diferencia de sexo no depende tanto de una diferencia anatómica sino de una distinción de capacidad relacional. No sólo las mujeres son las únicas capaces de ser activas en una relación sino que son ellas las que transmiten la aptitud para un hombre de tener un papel activo cuando se vuelven madres de los sobrinos y sobrinas de sus hermanos.

Traducido del francés por Julie-Jill Giffard

Horizontes Antropológicos, Porto Alegre, ano 16, n. 34, p. 405-426, jul./dez. 2010 


\section{Referencias}

BARNES, J. A. African models in the New Guinea Highlands. Man, n. 62, p. 5-9, 1962.

BONNEMÈRE, P. Le pandanus rouge: corps, différence des sexes et parenté chez les Ankave-Anga. Paris: CNRS Éditions; Éditions de la MSH, 1996.

BONNEMÈRE, P. Quand les hommes répliquent une gestation: une analyse des représentations et des rites de la croissance et de la maturation des garçons chez les Ankave-Anga (Papouasie Nouvelle-Guinée). In: GODELIER, M.; PANOFF, M. (Ed.). La production du corps: approches anthropologiques et historiques. Amsterdam: Editions des Archives Contemporaines, 1998. p. 81-113.

BONNEMÈRE, P. (Ed.). Women as unseen characters: male ritual in Papua New Guinea. Philadelphia: University of Pennsylvania Press, 2004.

BONNEMÈRE, P. Making parents: first-birth ritual among the AnkaveAnga of Papua New Guinea. Australian Religion Studies Review, v. 22, n. 2, p. 214-236, 2009.

BONNEMÈRE, P.; LEMONNIER, P. Les tambours de l'oubli: la vie ordinaire et cérémonielle d'un peuple forestier de Papouasie / Drumming to forget: ordinary life and ceremonies among a Papua New Guinea group of forest-dwellers. Papeete: Au Vent des Iles; Musée du Quai Branly, 2007.

DOJA, A. Rethinking the couvade. Anthropological Quarterly, v. 78, n. 4, p. 917-950, 2005.

DOUGLAS, M. The relevance of tribal studies. Journal of Psychosomatic Research, n. 12, p. 21-28, 1968.

FITI-SINCLAIR, R. Childbirth in Papua New Guinean villages and in Port Moresby General Hospital. In: LUKERE, V.; JOLLY, M. (Ed.). Birthing in the Pacific: beyond tradition and modernity? Honolulu: University of Hawai'i Press, 2002. p. 56-78. 
FORTES, M. The structure of unilineal descent groups. American Anthropologist, v. 55, n. 1, p. 17-41, 1953.

FRAZER, J. G. Totemism and exogamy: a treatise on certain early forms of superstition and society: v. 4. London: Macmillan and Co, 1910.

LÉVI-STRAUSS, C. La pensée sauvage. Paris: Plon, 1962.

LIPUMA, E. The gift of kinship. Cambridge: Cambridge University Press, 1988.

MALINOWSKI, B. Sex and repression in a savage society. London: Routledge, 1927.

MENGET, P. Temps de naître, temps d'être: la couvade. In: M. IZARD, M.; SMITH, P. (Ed.). La fonction symbolique: essais d'anthropologie. Paris: Gallimard, 1979. p. 245-264. (Bibliothèque des Sciences Humaines).

MUNROE, R. L., MUNROE, R. H.; WHITING, J. W. M. The couvade: a psychological analysis. Ethos, n. 1, p. 30-74, 1973.

POOLE, F. J. P. Couvade and clinic in a New Guinea society: birth among the Bimin-Kuskusmin. In: DE VRIES, M. W.; BERG, R. L.; LIPKIN JR., M. (Ed.). The use and abuse of medicine. New York: Praeger Publishers, 1982. p. 54-95.

RIVAL, L. Androgynous parents and guest children: the Huaorani couvade. Journal of the Royal Anthropological Institute, v. 4, n. 4, p. 619-642, 1998.

RIVIÈRE, P. G. The couvade: a problem reborn. Man, n. 9, p. 423-435, 1974.

STRATHERN, A. J. Kinship, descent, and locality: some New Guinea examples. In: GOODY, J. (Ed.). The character of kinship. Cambridge: Cambridge University Press, 1973. p. 21-34.

STRATHERN, M. The gender of the gift: problems with women and problems with society. Berkeley: University of California Press, 1988. 
THÉRY, I.; BONNEMÈRE, P. (Ed.). Ce que le genre fait aux personnes. Marseille: Editions de l'EHESS, 2008.

WAGNER, R. The curse of souw: principles of Daribi clan definition and alliance in New Guinea. Chicago: The University of Chicago Press, 1967.

Recebido em: 28/02/2010

Aprovado em: 06/07/2010 